mort, entre l'humanité et la bestialité. II est un être humain qui a basculé dans un entre-deux suite à une infection ou un envoûtement que seuls des rituels de purification, des rituels occultes et sacrés pourraient éventuellement ramener vers l'ordre présent dans le Vivant.[1,4]

L'esthétique du zombie est bien documentée dans l'univers des sciences sociales, par exemple en anthropologie par la pratique du Vaudou, en étude cinématographique et dans la littérature. Néanmoins, elle a été très peu explorée en sciences de la santé et pas du tout abordée ou presque en sciences infirmières. Une recherche dans CINAHL avec les mots-clefs zombies et nursing ont généré huit publications abordant différentes thématiques dont l'infection virale à Solanum[5], la zombie catégorie en santé mentale[6], les habits d'infirmières zombies sexy[7], l'apprentissage par simulation pour prévenir une infection[8] et le rôle des infirmières dans les films de zombies[9]. L'esthétique du zombie est donc quasi absente de la littérature scientifique infirmière. Pourtant, cette image du zombie peut nous en dire beaucoup sur qui nous sommes en tant qu'infirmières évoluant dans un espace sociopolitique.

Dans ce contexte, l'objectif de cette réflexion théorique critique est d'explorer l'esthétique du zombie en sciences infirmières.

\section{Le zombie}

Le zombie comprend différentes dimensions qui seront explorées successivement. En premier lieu, le corps du zombie, sa représentation au cinéma et dans les jeux vidéo, la charogne du zombie, sa monstruosité et la zombie catégorie. Les origines du zombie seront examinées en termes d'envoûtement et d'infection. Finalement, nous aborderons la question critique du patient zombie et de l'infirmière zombie.

\section{Le zombie est d'abord un corps}

Le corps de zombie est particulier. Ce corps révèle l'intérieur physique de la personne, les muscles, les tendons comme le tableau de Jean-Antoine Houdon daté de 1767, L'Écorché.[10 p33] Néanmoins, le zombie n'est pas aseptisé à l'instar d'une planche anatomique ou d'un travail de dissection médical.

Le zombie est un humain, un mort-vivant, dont les limites, les contours sont déchirés. Éventrés, éviscérés, ces corps déambulent les tripes à l'air, visibles aux yeux de tous, comme dans l'Enfer de Dante, tableau de Gustave Doré daté de 1861-1865.[10 p84] II comporte cette dimension Dantesque, apocalyptique qui terrorise, et est associée à la maladie et à la punition divine.

Le zombie est un corps en mouvement, car il est d'abord et avant tout un corps errant. II ne marche pas seul, mais se déplace en horde et constitue donc un danger apocalyptique, un risque pour tous les autres êtres humains à la fois car il est anthropophage,[2] mais aussi parce qu'il contamine les personnes non infectées : il zombifie.

\section{Le zombie au cinéma et dans les jeux vidéo}

Les œuvres cinématographiques mettant en scène des zombies sont innombrables, tout comme les ouvrages à leur sujet[11,12,2,13,14,15,16,9] II s'agit d'une industrie prospère qui critique la société, tel que nous le verrons plus loin, mais qui est aussi un objet de consommation.[15] Citons quelques exemples d'œuvres les plus connues : La Nuit des morts-vivants de G.A. Romero (1968); World War Z de M. Foster (2013) ou encore la télésérie The Walking Dead de F. Darabont (2010). Dans la quasi-totalité des œuvres cinématographiques, le zombie incarne un monstre sanguinaire très résistant qu'il faut éradiquer, sauf dans l'œuvre Warm Bodies de J. Levine (2013) qui décrit une romance entre une humaine et un zombie.[11]

Le rôle des infirmières au sein de la filmographie de zombie a été décrit par Stanley, Stanley et Magee en 2019.[9] Ces auteurs soulignent que seuls 10 des 485 films abordant les zombies mettent en scène des infirmières ayant un rôle de premier plan. Ces infirmières sont " dépeintes de manière romantique, sexualisées, caring, auto-sacrificielles et fortes ou démoniaques ".[trad. Lib. 9 p1751] Ils concluent que " peu de films mettent en scène des infirmières ayant des rôles centraux. Toutefois, la nature négative, violente et privée de ce genre offre une pauvre réflexion sur les sciences infirmières ". [trad. Lib. 9 p1751] Les œuvres cinématographiques mettent donc en scène des zombies sanguinaires, dynamiques, tantôt rapides, tantôt lents, mais mortels pour les vivants. Les infirmières y jouent des rôles stéréotypés.

Les jeux vidéo qui touchent au zombie sont également nombreux et populaires[17], tels que Resident Evil de Capcom et Virgin Interactive (depuis 1996); The Walking Dead de TellTale Games (2012); Left 4 Dead de Valve et Electronics Arts (2009) ou State of Decay de Undead Labs et Microsoft Studio (2013). Dans de nombreux jeux, le joueur a pour mission d'éradiquer les zombies au travers d'un scénario dynamique qui implique de la violence : décapitation, énucléation oculaire, fracture du crâne, explosion de cervelle, etc.

Les œuvres cinématographiques et de jeux vidéo s'interalimentent, puisque plusieurs titres couvrent des histoires similaires, tels que Resident Evil le film et les jeux Resident Evil. Le point commun entre ces deux industries, outre les prouesses visuelles, est certainement le spectateur et le joueur. Tous deux sont zombifiés au contact de ce type 
d'œuvre. En effet, leurs bestialités peut s'exprimer par la terreur, mais aussi, comme le souligne Coulombe, le plaisir de se repaître de scènes sanguinaires[16] qui jouent sur l'imaginaire humain.

L'image du zombie dans les œuvres populaires nous force à penser autrement, à " penser par l'horreur " [16 p3], par la laideur[18], par la mort[15] et par la charogne des corps morts-vivants putréfiés[3] à éliminer.

\section{Zombie et charogne}

La charogne est reliée à la mort des corps[3], à la puanteur incontournable de la décomposition des corps des zombies[19] et à l'abject[16]. C'est toute une activité grouillante de vie qui s'affaire à décomposer les corps organiques des animaux, êtres humains compris[3].

Afeissa qualifie l'esthétique de la charogne de "réalité des processus naturels de décomposition dans ce qu'ils ont de plus cru et d'insoutenable à la vue comme à l'odorat ".[3 $p$ 27] La charogne est rattachée aux liquides biologiques qui, au fil des heures, s'écoulent des corps morts.[3] II s'agit d'une forme de mort humide, une voie humide alchimique de transformation[20], de décomposition liée aux sucs et microorganismes qui se repaissent des corps morts[3].

La charogne est intimement liée à l'état de décomposition du corps du zombie. Selon Le Maître[2], les zombies constituent une fiction du cadavre que l'on sera un jour. Ainsi, "l'odeur du zombie est un rappel de notre mortalité, vécue comme une dualité poignante : l'odeur du zombie qui approche nous rappelle notre mort par l'expérience de l'abject, et le zombie, en tant que monstre, a le pouvoir d'actualiser la mort ".[19 p207-8]

L'esthétique du zombie " fait rupture (...) rompt avec notre société hygiéniste et obsédée par le contrôle des corps, des odeurs, des signes de vieillesse, une société où la mort est désormais taboue. (...) paradoxe de l'horreur, ces corps et cette mort éveillent notre curiosité et nous rappellent ce que nous préférons ne pas voir ".[16 p78]

Les infirmières semblent être peu en contact avec la charogne, avec cette décomposition humide cadavérique. Elles font plutôt face aux corps morts aseptisés, souvent encore intacts, chauds, et qui se refroidissent. II s'agit d'une forme de mort sèche[20] où la décomposition sera suspendue dans le temps, temporairement, puisque le corps sera rapidement envoyé à la morgue et préservé par le froid en attendant que les rituels mortuaires puissent s'opérer.

Néanmoins, dans leur pratique, les infirmières entrent en contact avec les liquides biologiques, mais habituellement pas ceux liés à la décomposition macabre du corps. Les infirmières hospitalières évoluent dans des milieux aseptisés sur le plan symbolique où la blancheur et l'ordre tentent d'être maintenus malgré le désordre imposé par la maladie et la noirceur de la mort. Pourtant, même si la charogne n'est pas apparente, les pathogènes sont prégnants dans les établissements de santé, mais sont invisibles, car microscopiques. Ces pathogènes sont parfois qualifiés de "bibites ", de monstres microscopiques.[21]

\section{La monstruosité du zombie}

Le zombie peut être, à bien des égards, vu comme un monstre qui fait appel à notre "imaginaire social ".[22] Toutefois, le zombie n'est pas un monstre habituel. II se situe à l'intersection entre le monde des vivants et des morts comme Frankenstein.[23] Les monstres, quant à eux, sont souvent soit vivants, tels les infirmes; morts, tels les fantômes ou issus de la mythologie.[22,10] Néanmoins, zombies et monstres ont en commun l'imaginaire, le monde fantasmagorique, possédant des traits humains ou humanoïdes. Zombies, géants maléfiques, ogres ou ogresses sont des humains qui dévorent d'autres humains.[10]

La notion de monstre est polysémique et polymorphe dans la temporalité. Pour pouvoir aboutir à une définition, il faudrait selon Caiozzo et Demartini " embrasser la totalité d'un champ qui s'avère singulièrement hétérogène, depuis les chimères de la mythologie gréco-latine jusqu'au fœtus cyclocéphaliens ou sirénomèles des musées d'anatomie pathologique, en passant par les grylles multicéphales des manuscrits médiévaux et par les femmes à barbe, hommes-squelettes et autres phénomènes des entre-sorts et tréteaux de foires " .[22 p10-11]

La monstruosité fait appel à un système de représentations sociales, à des seuils de sensibilité; elle est liée à la déviance criminelle, à l'infirmité du corps qui peut être corrigée parfois par la chirurgie.[22,23] Cette infirmité du corps, ce handicap place le monstre à risque au sein de la société qui peut être vu comme un malheur, un déséquilibre, un signe de sorcellerie[24], une catastrophe ou le résultat d'une infection[25]. Quoiqu'il en soit, l'infirmité, le handicap, le hors norme se situe entre ombres et lumières[26].

L'image du monstre est incarnée aussi par les hommes et les femmes tatoués sur l'ensemble de leurs corps qui offraient des représentations dans les foires et expositions.[27] Un écho contemporain à cette attraction foraine est le personnage de Zombie Boy incarné par Rick Genest.[28] Ce type de tatouage 
zombie en tant qu'art corporel, esthétique du corps, mêle fascination et répulsion, ordre et désordre, santé et folie. Bien que le tatouage soit " une réalité anthropologique, un phénomène social, une production artistique "[27 p203], il demeure associé aux tabous[27] et à la transgression des normes sociales, particulièrement quand ces derniers couvrent l'ensemble du corps à l'instar de Zombie Boy. Le tatouage intégral de Zombie Boy peut incarner un symbole d'autodestruction pour la personne elle-même, mais constituer un risque d'autodestruction pour la société également, car déviant de la norme et donc dangereux, monstrueux.

\section{La zombie catégorie}

Si le monstre, le zombie constitue un risque pour la société, la zombie catégorie d'Ulrich Beck l'explique de manière éloquente et frappante. En effet, les travaux de Beck sur la société du risque[29] ont évolué vers ce qu'il appelle à présent la zombie catégorie[30]. Selon Beck, la zombie catégorie est un espace de risque, une catégorie morte-vivante telles que " la famille, la classe sociale, le voisinage ".[30 p205] Ce sont autant de catégories qui se désintègrent selon lui, des lieux de tensions entre les hommes et les femmes, des lieux de conflits et d'expression des iniquités. À titre d'exemple de catégorie zombie, Beck propose le " plein emploi ". II le décrit comme le modèle d'après-guerre d'aide sociale présentant un faible taux de chômage, incarné par un père pourvoyeur issu de la classe-moyenne qui conservera un emploi stable à vie.[30] II oppose ce modèle à des emplois temporaires, flexibles, à temps partiel qui caractérisent davantage la société contemporaine individualisée.[30] Le plein emploi est donc une catégorie zombie, soit une catégorie morte-vivante.

Gross approfondit la notion de catégorie zombie en soulignant que le risque fait appel à l'inconnu, mais que la notion même de risque pourrait "être substitué à la non-connaissance ".[31 p386] Cette non-connaissance constitue justement la zombie catégorie.[31] Afin d'illustrer la zombie catégorie Gross recourt à des exemples rattachés au terrorisme et à la santé. Selon Gross, en cas de menace terroriste, la zombie catégorie est composée de toutes ces informations non divulguées au public lorsqu'un risque d'attentat surgit, mais aussi de ce que les autorités ne savent pas, soit de l'inconnu.[31] Au niveau de la santé, Gross recourt à l'exemple des identités génétiques pour illustrer la zombie catégorie. Selon lui, tester les gènes et tenter d'identifier le potentiel que des maladies se développent repose sur un calcul de probabilité dépourvu de sens qui en dit plus long sur la non-connaissance que sur la connaissance elle-même d'une situation donnée.[31]

Reconnaître la zombie catégorie tel que le soutient Gross, c'est aussi reconnaître l'existence de nos limitations humaines face aux attaques terroristes, à nos potentiels génétiques[31], à la santé mentale[6] ou aux infections. Le zombie et la zombie catégorie incarnent donc toutes ces peurs d'un futur incertain, d'un risque, d'un danger pour l'Humanité.

\section{Les origines du zombie : envoûtement et infection}

Le zombie est un mort-vivant qui ne se situe pas seulement dans l'imaginaire humain, création monstrueuse de nos esprits, incarnation de nos passions et de nos peurs.[14] Cette image du zombie prend sa source dans la matérialité de la vie humaine. La zombification est ancrée à la fois dans le monde matériel et dans le monde métaphysique. Elle peut être causée par des pathogènes[5], des drogues, ou par un envoûtement induit par un sorcier[2]. Afin de prévenir la zombification, les êtres humains peuvent se faire désenvoûter par un rituel de purification, et recourir à la prévention des infections, tel que nous l'aborderons ici.

\section{Envoûtement - désenvoûtement et politique}

Le phénomène zombie prend sa source dans les Antilles, particulièrement en Haïti[1,4], mais il est présent à des degrés différents en Amérique Latine, surtout dans les Caraïbes[32]. La personne pourrait paraître envoûtée soit par l'usage d'une drogue, soit par l'ensorcellement.

La personne sous influence d'une drogue zombifiante[1], la drogue Zombie, burundanga, souffle du diable ou drogue du voleur, erre comme un zombie. II s'agit de scopolamine, une drogue ayant des effets hallucinogènes et qui peut provoquer une amnésie.[33]

La personne peut aussi être victime d'une zombification, d'un envoûtement causé par un sorcier, cet Autre qui nous amène dans un état de conscience altérée, une forme de transe.[2,15] Si la zombification est provoquée par un ensorcellement, cette dernière peut sortir de cet état de conscience altérée par un désenvoûtement vaudou en pratiquant un rituel secret[4] opéré par une personne ayant des pouvoirs magiques[1].

Selon Saint-Gérard, les personnes victimes de zombification et qui sont en transes, investies par des esprits, sont souvent des domestiques qui peuvent retrouver la parole[1] lors du rituel désenvoûtement. Leur glossolalie entremêlée de discours cohérents est " rattaché à une persécution imaginaire par d'autres ".[1 p66]

Toutefois, cette persécution n'est pas si imaginaire que cela. En effet, il faut tenir compte du contexte sociohistorique de la colonisation des Antilles et des Caraïbes ainsi que du contexte sociopolitique pour comprendre l'émergence du phénomène zombie. 
Certains auteurs latino-américains soutiennent que le phénomène zombie est lié à la colonisation de l'Amérique Latine[32], car le " zombie (est un) symbole douloureux de l'esclavage afro-antillais "[Trad. Lib. 24 p135]

Saint-Gérard, quant à lui, souligne que " au-delà de l'imaginaire, le politique se faufile dans les interstices du vodou pour rationaliser les aberrations d'une dictature rétrograde vieille de près de deux siècles (en Haïti). Soupape de sécurité, le vodou canaliserait ainsi les mécontentements sociaux à travers les pseudo-rivalités d'individus privés de leur droit élémentaire de la parole ".[1 p72]

Plus largement, la société de consommation[35] provoque une zombification, un envoûtement par la consommation de produits et de services au sein d'une société capitaliste. Harman parle même d'un capitalisme zombie[36]. Un symbole actuel de zombification serait les utilisateurs de téléphone intelligent qui errent dans les rues, davantage préoccupés par leurs écrans que par leur environnement physique et social direct qui les entourent. Dans un contexte globalisé, il paraît plus difficile de se désenvoûter, de sortir de cette transe collective de consommation.

Si l'on considère une perspective anthropologique plus individuelle, seuls des rituels de purification par des processus occultes[4] comprenant aussi des chants et l'utilisation de divers objets de culte vaudou[2] permettent de désenvoûter le zombie en tentant de le ramener vers les Vivants. Si le désenvoûtement échoue, le rituel mortuaire devient essentiel. "La mort est un rituel social (...) ultimement, le rituel confirme la place qu'occupe la personne au sein de la fabrique de la vie- la vie de leur famille, de leur communauté, et de leur monde ".[trad. Lib. 37 p235] Avec le mort-vivant, le rituel mortuaire ne peut justement pas s'opérer. On ne peut pas laisser le zombie confiné à un entre-deux : il doit être soit vivant, soit mort, sinon il ne peut prendre sa place dans l'ordre de la société, et créera le désordre en contaminant le vivant.

\section{L'infection à Solanum}

La zombification peut être théoriquement causée par une infection à Solanum (virus Z) qui pourrait provoquer une éclosion, voire une épidémie zombie.[5] Dans le cadre d'une zombification, l'infection se propage à l'humain souvent par la morsure d'un zombie.[2]

Stanley mentionne que "le virus entre dans le système sanguin de la victime après une morsure ou un contact avec les fluides corporels d'un zombie contaminé (les sources principales de contamination sont le sang ou les produits sanguins, la salive, les vomissures et les expectorations) ".[trad. Lib. 5 p1607]
Cette contamination fantasmagorique au virus $Z$ est similaire à la transmission réelle de la rage,[38] une zoonose virale qui frappe également l'imaginaire par la bestialité et l'agressivité du chien infecté, salivant, qui mord son humain.

Tout comme pour l'envoûtement, dans la zombification par transmission d'une infection, c'est toujours cet Autre qui est responsable de la contamination, du sort, du maléfice, de l'infection.

\section{La prévention de l'Infection}

La prévention des infections fait appel à un rituel de purification selon Douglas[39], mais fondé sur la science. Le rituel va contribuer à ramener le corps du zombie vers le vivant, car on tente de prévenir l'infection, d'éviter qu'elle se propage, voir la traiter.

Les infirmières jouent un rôle important en prévention des infections.[40] Elles travaillent en collaboration avec d'autres professionnels de la santé ainsi qu'avec les patientspartenaires de soins, les familles et les visiteurs afin de gérer les risques reliés aux infections.

Cette gestion des risques passe aussi par le recours à des mesures de prévention des infections comme les mesures de base, telles que le lavage de mains ou les précautions additionnelles comme l'isolement des patients infectés et le port d'équipement de protection individuel (masque, blouse, gants, visière, etc.).[41,42]

Les infirmières ont également un rôle de surveillance à exercer afin de maîtriser les infections et de les documenter à l'aide de statistiques populationnelles pour mieux pouvoir les combattre.[21] Elles contribuent à la prévention des infections sur le plan local, régional, provincial et national en brisant la chaîne de transmission des infections.[43] Elles contribuent à maintenir l'ordre et à repousser I'Infection, la maladie synonyme de désordres des corps physique et social.

\section{Le patient et l'infirmière zombies}

Les patients infectés sont susceptibles de se transformer en zombie lorsque les infirmières n'ont pu contenir l'infection par les mesures de prévention des infections à l'intérieur de l'hôpital en les isolant de manière systématique et ordonnée pour prévention la propagation de l'infection à toute la population.[5] L'Infection pandémique se répandrait au travers de la société et les patients erreraient dans les villes tels des morts-vivants. Ils seraient livrés à eux-mêmes et envahiraient les rues des villes en constituant une menace au Vivant dans un espace empreint de désordre et de multitude, de horde sauvage, car les zombies se multiplient "comme de 
la vermine ".[13 p9]

L'infirmière quant à elle, combat l'Infection provoquée par cet Autre qui repousse et génère le désordre. Toutefois, l'infirmière elle-même est susceptible de tomber dans le chaos et de devenir zombie elle-même. La zombification de l'infirmière passe par la contamination de son corps et de son esprit, car cette dernière est à risque de contracter elle-même une infection au virus $Z$ en cas d'éclosion ou de pandémie zombie. En effet, tel que Stanley le souligne, l'infirmière est susceptible de contribuer à " des amputations de membres, de procéder à des isolements de patients, à gérer les infections, à évaluer les risques à la sécurité, à soigner des plaies, à soulager la douleur et la fièvre par l'administration de médicaments ".[trad.lib. 5 p1610] Elle travaillerait donc au contact de personnes infectées par le virus $Z$ hautement contagieux.

La zombification de l'infirmière passe aussi plus globalement par la nature assujettissante et violente de son travail. En effet, tel que le souligne Lakeman et Molloy, le système de santé a "besoin de travailleurs serviles et non critiques, (et favorise) une pratique dirigée par des protocoles " [trad.lib. 6 p1009], ce qui encourage une non-pensée. Ils constatent également un renforcement de ce qu'ils nomment l'hégémonie médicale dans les espaces de pratique des infirmières.

La zombification de l'infirmière passe également par l'enseignement-apprentissage théorique durant la formation. En effet, Foucault souligne que l'école est un espace de dressement des corps au même titre que la prison ou l'asile.[44] C'est un lieu d'apprentissage où l'enseignement assujettit autant qu'il libère. Si l'école assujettit, nous soutenons aussi qu'elle zombifie à l'instar de Lakeman et Molloy qui n'hésitent pas à parler de zombification du monde académique.[6] Cette zombification passe par la création d'une catégorie zombie lors de l'apprentissage théorique en sciences infirmières.

La quasi-totalité des écrits théoriques classiques contemporains en sciences infirmières mettent le corps humain à distance. Seule l'école des besoins dont les écrits de Virginia Henderson font partie, aborde les fonctions vitales du corps comme le besoin de respirer.[45] Récemment, Vonarx plaidait pour l'introduction du corps au sein du métaparadigme infirmier comme concept central de la discipline.[46] Quoiqu'il en soit, le registre des discours scientifiques en sciences infirmières abordent très rarement la question des odeurs, de la putréfaction, de la charogne, des viscères, voir des couleurs associées aux corps. Les seules couleurs ou textures qui paraissent être enseignées sont celles des excréments, excrétions ou des téguments afin de vérifier indirectement certaines fonctions corporelles.[47] Ce qui semble plutôt valorisé est la technicité et la pureté du soin à la fois en termes d'asepsie et d'antisepsie, mais aussi sur le plan symbolique de la pureté.[39]

Le corps humain est invisibilisé, alors que l'infirmière possède elle-même un corps et les personnes qu'elles soignent également. Les concepts utilisés en sciences infirmières font appels à la personne, à son environnement, à sa santé et aux soins selon le métaparadigme qui se décline différemment selon les théoriciennes.[45] Certaines théories sont idéalistes, dont le caring,[48,49] spirituelles[50] ou constituent à elles-seules des zombies catégories en parlant d'être-humain-en-devenir[51]. En effet, comment théoriser la non-connaissance [31] d'un être-humain-en-devenir[51], ce potentiel, ce risque non encore avéré? Ces théories idéalistes en sciences infirmières semblent s'opposer à un matérialisme philosophique plus près des corps et de l'existence humaine.[52,53]

À notre connaissance, aucune théorie classique reconnue semble aborder le corps humain, la personne, en tant que mammifère inclus dans le règne animal. La plupart des théories dématérialisent, effacent les corps, invisibilisent la nature charnelle humaine, et misent sur des concepts ou idéaux moraux. Nous pensons que ceci a pour effet de nier la nature humaine et de renforcer le fossé entre théories académiques et pratiques cliniques.

L'image de l'infirmière zombie vient rappeler aux théoriciennes la nature charnelle des corps, l'inscription des expériences vitales, viscérales dans ce corps soigné. Cette figure de l'infirmière zombie transgresse les tabous et peut sembler se situer dans la marge, être hors-norme. II n'en est rien : l'infirmière zombifiée est chacune d'entre nous, errant à l'aveugle, d'abord confinée à nos quarts de travail pour ensuite contaminer tous nos espaces de vie. Comme le dit Jean Clair, " le monstre a fini par tout envahir ".[10 p167] L'infirmière zombie est un mode de survie, ni tout à fait morte, ni tout à fait vivante. Cela nous amène à nous demander comment les infirmières peuvent-elles, collectivement, sortir de cet état de zombification?

\section{Conclusion}

L'esthétique du zombie en sciences infirmières est quasi absente de la littérature scientifique. Pourtant, elle est parlante car l'infirmière peut-être à la fois celle qui combat les infections, mais être infectée à son tour dans le cadre de son travail. L'infirmière est à la fois agent qui ordonne, confine, isole pour prévenir l'infection, mais elle est aussi zombifiée par son travail tant par la nature des soins qui sont exigés au 
contact avec les patients que par sa formation académique.

Un approfondissement de cette réflexion théorique critique serait nécessaire par différentes pistes de recherche. Par exemple, l'étude de la présence des infirmières et des rôles qu'elles jouent non seulement dans les domaines cinématographiques tel qu'étudié par Stanley et al.[9], mais aussi du jeu vidéo et littéraires lorsque des zombies sont mis en scène. Ou encore l'exploration plus approfondie des dimensions anthropologiques du Zombie et des ponts à établir avec les sciences infirmières. Finalement, quelques questions s'imposent: comment les infirmières font-elles face aux monstres, aux zombies et à tout ce qui est hors norme? ; comment les infirmières théoriciennes peuvent produire autre chose que des zombies catégories? ; comment dézombifier le monde académique en sciences infirmières?

\section{References}

1.Saint-Gérard Y. Le phénomène zombi: la présence en Haïti de sujets en état de non-être. Toulouse: Erès; 1992.

2.Le Maître B. Zombie, une fable anthropologique. Paris: Presses Universitaires Paris Ouest; 2016.

3.Afeissa H-S. Esthétique de la charogne. Paris: Éditions Dehors; 2018.

4.Salgado A. Le Phénomène des Zombis dans la Culture Haïtienne. Antilles: Imprimerie des Antilles; 1982.

5.Stanley D. The nurses' role in the prevention of Solanum infection: dealing with a zombie epidemic. Journal of Clinical Nursing. 2012;21(11-12):1606-13.

6.Lakeman R, Molloy L. Rise of the zombie institution, the failure of mental health nursing leadership, and mental health nursing as a zombie category. International Journal of Mental Health Nursing. 2018;27(3):1009-14.

7.Brown SA. On "Nurse Zombie" and Sexy Nurse Themed Fancy Dress Costumes. Nursing Standard. 2015 Nov 4;30(10):3131.

8.Claus N, Powers S, Wingo NP. Using Simulation and TeamSTEPPS to Reinforce Nonvalidated Skills: "The Zombie Sim.” Journal of Nursing Education. 2019 Feb 5;58(2):124124.

9.Stanley D, Stanley K, Magee D. Celluloid zombies: A research study of nurses in zombie-focused feature films. Journal of Advanced Nursing. 2019;75(8):1751-63.

10.Clair J. Hubris: la fabrique du monstre dans l'art moderne: homoncules, géants et acéphales. Paris: Gallimard; 2012.
11.Inguanzo O. Les Zombies au cinéma: L'Histoire ultime des morts-vivants à l'écran. Paris: Hoebeke; 2017.

12.Boluk S, Lenz W. Generation Zombie. Essays on the Living Dead in Modern Culture. Jefferson: McFarland; 2011. 259 p.

13.Perron B, Domínguez Leiva A, Archibald S. Z pour zombies Montréal: Les Presses de I'Université de Montréal; 2015.

14.Pepin A. Zombie: le mort-vivant autopsié. Québec: Les Intouchables; 2013.

15.Balaji M. Thinking dead: what the zombie apocalypse means. Lanham: Lexington Books; 2013.

16.Coulombe M. Petite philosophie du zombie ou comment penser par l'horreur. Paris: Presses Universitaires de France; 2012.

17.Maheux F. Du vaste à l'exigu: l'invasion du zombie dans les espaces vidéoludiques. In: Z pour Zombies. Montréal: Presses de l'Université de Montréal; 2015. p. 189-201.

18.Ribon M. Archipel de la laideur: essai sur l'art et la laideur. Paris: Kimé; 1995.

19. Niedenthal S. Un doux parfum de dégoût. L'avenir du zombie à l'ère du jeu vidéo odorant. In: Z pour Zombies. Montréal: Presses de l'Université de Montréal; 2015. p. 20315.

20.Von Franz M-L. Alchimie - Une introduction au symbolisme et à la psychologie. Paris: La Fontaine de Pierre; 2000.

21.Bernard L. Analyse critique de la culture de sécurité face aux risques biologiques et pandémiques pour les infirmières. Université de Montréal; 2012.

22.Caiozzo A, Demartini A-E, Ancet P. Monstre et imaginaire social: approches historiques. Paris: Créaphis; 2008.

23.Davison CM, Mulvey-Roberts M. Global Frankenstein Cham: Springer; 2018.

24.Gardou C. Le handicap au risque des cultures. Paris: ERES 2010.

25.Giuliani G. Monsters, Catastrophes and the Anthropocene : A Postcolonial Critique. Londres: Routledge; 2020.

26. Marques S. Au Brésil, le handicap en ombres et lumières. Paris: ERES; 2010.

27.Martin L. Tatouages et tabous. Sociétés Représentations. 2016;42(2):201-3.

28.ARTE. Documentaire: Zombie Boy. 2019.

29.Beck U. La Société du risque: Sur la voie d'une autre 
modernité. Paris: Aubier; 2001.

30.Beck U, Beck-Gernsheim E. Individualization: institutionalized individualism and its social and political consequences. London; Thousand Oaks: SAGE; 2001.

31.Gross M. Risk as zombie category: Ulrich Beck's unfinished project of the 'non-knowledge' society. Security Dialogue. 2016;47(5):386-402.

32.Jauregui CA, Dabove JP. Heterotropias: narrativas de identidad y alteridad latinoamericana. Pittsburgh: Instituto Internacional de Literatura Iberoamericana, Universidad de Pittsburgh; 2003.

33.World Health Organization. WHO Model lists of essentials medicines. WHO; 2013.

34.Aizenberg E. "I walked with a zombi" placeres y peligros de la hibridez poscolonial. In: Heterotropias: narrativas de identidad y alteridad latinoamericana. Pittsburgh: Instituto Internacional de Literatura Iberoamericana, Universidad de Pittsburgh; 2003. p. 135-47.

35.Beaudrillard J. La société de consommation. Paris: Gallimard; 1974.

36. Harman C. Zombie capitalism. Chicago: Haymarket Books; 2010.

37.Schultz E, Lavenda R, Dods R. Cultural anthropology. Fourth Canadian Edition. 4th ed. New York: Oxford; 2018.

38.Ministère de la santé et des services sociaux du Québec. Rage chez I'humain [Internet]. 2019. Available from: https:// www.quebec.ca/sante/problemes-de-sante/a-z/rage-chezhumain/

39.Douglas M. De la souillure. Paris: La Découverte; 2005.

40.Ordre des infirmières et infirmiers du Québec. Protéger la population par la prévention et le contrôle des infections: une contribution essentielle de l'infirmière. [Internet]. 2008. Available from: https://www.oiiq.org/uploads/publications/ autres_publications/237_prevention.pdf

41. Létourneau J, Alderson M, Leibing A. Positive deviance and hand hygiene of nurses in a Quebec hospital: What can we learn from the best? American Journal of Infection Control. 2018;46(5):558-63.

42. Mayhall C. Hospital Epidemiology and Infection Control. Wolters Kluwer; 2011.

43.Nies M, McEwen M. Community/Public Health Nursing: Promoting the Health of Populations. 7th ed. St. Louis: Elsevier; 2019.
44.Foucault M. Surveiller et punir. Paris: Gallimard; 1975.

45.Pepin J, Ducharme F, Kérouac S. La pensée infirmière. 4th ed. Montréal: Chenelière; 2017.

46.Vonarx N. Le corps au sein de la discipline infirmière: un incontournable pour lier les quatre concepts du métaparadigme actuel. Recherche en Soins Infirmiers. 2016;4(127):8-15.

47.Potter P, Perry A, Stockert P, Hall A. Soins infirmiers: Fondements généraux. 4th ed. Montréal: Chenelière; 2016.

48.Cara C, Gauvin-Lepage J, Lefebvre H, Létourneau D, Casimir $\mathrm{M}$, Alderson $\mathrm{M}$, et al. Le modèle humaniste de soins infirmiersUdeM: perspective novatrice et pragmatique. Recherche en soins infirmiers. 2016;2(125):20-31.

49.Ray M. Consciousness and the moral ideal: a transcultural analysis of Watson's theory of transpersonal caring. Advanced Practice Nursing Quarterly. 1997;3(1):25-31.

50. Hektor LM. Martha E. Rogers: a life history. Nursing Science Quarterly. 1989;2(2):63-73.

51.Parse RM. L'humain en devenir. Nouvelle approche du soin et de la qualité de vie. Québec: PUL; 2003.

52.Onfray M. L'art de jouir : Pour un matérialisme hédoniste. Paris: Grasset; 1991.

53.Politzer G. Principes élémentaires de philosophie. Paris: Delga; 2009.

Remerciements

Aucun financement n'a été reçu pour rédiger cet article théorique. Aucun conflit d'intérêt connu n'est à déclarer. Nous tenons à remercier Dre Agnès Bernard et Madame Myriam Rosseau pour les nombreux échanges sur les infirmières zombies. Nous remercions également la Pre Annette Leibing de nous avoir pistée sur les catégories de zombies afin d'approfondir notre réflexion.

Pour contacter l'auteure:

Laurence Bernard, inf. Ph.D.,

Professeure agrégée

Université de Montréal

Faculté des sciences infirmières

C.P. 6128 succ. Centre-Ville

Montréal, Québec, H3C 3J7

Canada

Courriel: Laurence.bernard@umontreal.ca 